\title{
PREDICTING VARIABLE STAR LIGHT CURVES
}

\author{
T. SERRE \\ DASGAL, Observatory of Paris-Meudon, URA CNRS 335, F.92195 Meudon, France
}

\begin{abstract}
I present a new method to test determinism, in particular the nonlinear behavior, in observed time series of pulsating stars, based on a recent prediction method which exploits the dynamical system theory. A method for filling gaps in data has thereby been constructed. Estimated bounds to the necessary embedding dimension can be obtained and chaotic divergences can be estimated.
\end{abstract}

\section{Principles of the Prediction Method}

This method uses a reconstructed representation of the signal $s(t)$, with vectors $\mathrm{X}(i \delta t)=(s(i \delta t), s(i \delta t+\tau), \ldots, s(i \delta t+(m-1) \tau))$ in a m-dimensional state space with $\tau=d \delta t$, the time delay and $\delta t$, the time step of the signal, by using the time delay method Takens (1980). The reconstructed attractor is equivalent to an attractor constructed with the true variables describing the dynamics of the underlying physical process. In order to faithfully reproduce the attractor, this reconstruction requires a sufficiently large number of observed cycles and low noise level, that depend on dynamics complexity.

The true local law, $\mathbf{F}$, in the neighbourhood of a point of an attractor, links a past vector $\mathbf{X}(i \delta t)$, to its future vectors $\mathbf{X}(i \delta t+T)$, with a time step $T$, i.e. $\mathbf{X}(i \delta t+T)=\mathbf{F}(\mathbf{X}(i \delta t))$. In this neighbourhood, the closest vectors (this learning set is found by fast sorting and searching methods), are used to perform a least square approximation $\mathbf{F}_{a}$ of $\mathbf{F}$, once the order of Taylor expansion for $F_{a}$ is fixed. This process is then iterated by estimating $F$ at each predicted point (Farmer and Sidorowich, 1988, see the review of Casdagli et al. 1991). The basic assumption of this method is that a deterministic behavior must be present to assure the existence of a stationary structure, i.e. an attractor, in the state space.

\section{Astrophysical Interests of This Method}

The above method enables to fill short gaps, which are seen as a missing trajectory in the attractor, in an observed signal. It can be of interest for signals resulting from multisite campaigns, by identifying aliases. It differs from the MEM (Maximum Entropy) Method (Fahlman and Ulrych 1982) mainly in the fact that it uses local fits: the predictions are made by interpolations on the reconstructed attractor and local extrapolations where the goodness is controled by using neighbor vectors, instead that MEM uses an ARMA predictor with global fits, which gives an extrapolation in the gaps, with statistical controls, based in an interpolation on the available signal. 
The method used here does not create information if a successful dynamics approximation on the known parts is performed in the limit of the prediction time. Random or unknown dynamics are not predictable if not contained in the available recorded signal. Applications on artificial signals are successful (Serre et al. 1992).

Applications in identifying aliases in RV Tauri star are successful, but are more difficult when applied to light curve of a $\delta$ Scuti star GX Peg. Successful aliases reduction on a model of this star ( 5 artificial frequencies) shows that the noise level is the principal limitation and further studies are needed.

This prediction method is also used for testing divergences in the chaotic attractors. The tests on Rössler chaotic attractor are successful. An application on a hydrodynamical model of type II cepheids (from Kovács and Buchler 1987) confirms his chaotic nature. A method for estimating the correct embedding dimension or a lower bound (the efficient embedding dimension), by testing the best predictions, is adapted (Serre and Buchler, 1992) from a method of Casdagli et al. (1991). A test on the RV Tauri star, $\mathrm{R}$ Scuti, has thereby given only a lower bound, 4 or 5 , for the embedding dimension, because of the stochastic perturbations.

\section{Conclusions}

This approach provides a new way of analyzing signals based on state space representation. The dynamics can be characterized by local or global modellings of the attractor (Casdagli et al., 1991). These modellings have a great potential for a deeper understanding of chaotic hydrodynamical models. Presently, the noise is the main limitation to the full efficiency of this method. Stochastic perturbations due to instrumental uncertainties and atmospheric noise must be reduced to low level by data processing methods preserving the dynamics information. A method, based on prediction, developed by Kostelich and Yorke (1988) is an interesting approach on noise reduction problems.

\section{Acknowledgements}

I thank Dr. D. Kurtz and Dr. W. Dziembowski for useful remarks during the colloquium, and, Dr. M. Auvergne, M. J. Goupil and J. R. Buchler for useful discussions about this work.

\section{References}

Casdagli, M., Des Jardins, D., Eubank S., et al.: 1991, Tech. Rep. LA-UR-91-1637 (Los Alamos Nat. Lab.)

Fahlman G. G. and Ulrych T. J.: 1982, Monthly Notices of the RAS 199, 53. 
Farmer J. D. and Sidorowich J. J.: 1988, Tech. Rep. LA-UR-88-901 (Los Alamos Nat. Lab.)

Fostelich E. J. and Yorke J. A.: 1990, Physica D 41, 183.

Kovács G. and Buchler J. R.: 1988, Astrophysical Journal 334, 971.

Serre, T., Auvergne, M.. and Goupil, M. J.: 1992, Astronomy and Astrophysics. in press.

Serre T. and Buchler J. R.: 1992, in preparation.

Takens F.: 1981, in Dynamical System and Turbulence, eds. Rand D. and Young L. S.(Springer Lecture Notes in Mathematics 898, Berlin), p. 366. 